\title{
Assessment of 3-year prognosis in survivors of acute myocardial infarction
}

\author{
C. Helmers \\ From the Department of Medicine at Serafimerlasarettet, Stockholm, Sweden
}

A 3-year follow-up with regard to survival was made of 475 initial survivors of an acute myocardial infarction originally treated in a coronary care unit. Previous diseases, complications during the hospital stay, as well as findings recorded on discharge from hospital, were used to construct a prognostic stratification. By multivariate analyses maximum respiratory rate during the coronary care unit stay, left bundle-branch block, and age proved prognostically most important.

Using only these factors, patient groups with a 3-year mortality up to 74 per cent were characterized.

The problem of evaluating prognosis is an important aspect of clinical medicine. In spite of the introduction of the coronary care unit the prognosis in hospital of patients with acute myocardial infarction is both dubious and difficult to foretell in the individual case. The difficulties of the long-term prognosis are no less though many studies have been concentrated on the prognostic implications of different clinical factors. As prognostic stratification is necessary as a basis for therapeutic trials some long-term prognostic indices and risk profiles have been published (Peel et al., 1962; Norris et al., 1970; Oxman et al., 1972; Vedin, 1974). To be of practical value such indices must be easy to apply and preferably include only a few factors which are objective. The present study is a continuation of a previous one which dealt with, mainly, the two-year prognosis in patients surviving an acute myocardial infarct (Helmers, 1973).

\section{Patients and methods}

From 1968 to 1970,475 patients with acute myocardial infarction were initially treated in the coronary care unit at Serafimerlasarettet and discharged alive. A description of the unit, criteria for admission and diagnosis, as well as definitions of different complications have been published (Biörck et al., 1969; Helmers, 1973). Relevant symptoms, physical findings, laboratory data, and complications in the coronary care unit as well as the remainder of the hospital period were recorded by code on special charts and transferred to punch cards. A three-year follow-up with regard to survival was made

Received 25 November 1974. of 466 patients, 307 men (66\%) and 159 women ( $34 \%$ ). The mean age in the patient group was 65 years on admission, range 39 to 93 years. Nine foreigners could not be traced and were excluded from the study. Deaths from all causes were recorded.

To create prognostic strata with as distinct clinical profiles as possible automatic interaction detector analysis (Sonquist and Morgan, 1964) was performed. Automatic interaction detector analysis as described in the Osiris Program instruction manual (I97I) is something like a 'stepwise regression programme'. Regarding one variable (e.g. death) as the dependent variable, the analysis employs a nonsymmetrical branching process, based on variance analysis techniques, to subdivide the sample into a series of subgroups which maximize one's ability to predict values of the dependent variable. The independent variables ('predictors') need not be quantitative. One can have either quantitative categories (as for age or income), or 'qualitative' categories (as for sex, marital status, cause of death, or political preference). In addition, with automatic interaction detection, the quantitative predictors can be classified into intervals of unequal length (e.g. incomes under 15000, 15000 to 24999,25000 to 49999 , etc.) and even into non-ordinal categories (e.g. ages 24 to 54,20 to 24 , or 55 to 64 , and 65 and over). Linearity and additivity assumptions inherent in conventional multiple regression techniques are not required. Groups with less than Io patients were not accepted in these analyses. For further illustration of the relations between selected clinical factors, stepwise linear regression analysis was also made (Biomedical Computer Program: BMD 02 R). The factors studied in the analyses have been presented in detail (Helmers, 1973) and a summary is given in the Table. As seen, blood lipids were not included as a factor. For testing the significance of differences of proportions the $\chi^{2}$ test was used 
$\mathrm{TABLE}$ Factors included in coronary care unit record and studied in the multivariate analyses

\begin{abstract}
Sex, age
Previous myocardial infarction, angina pectoris, hypertension, heart failure, diabetes, smoking habits

Coronary care unit stay: Signs of heart failure (pulmonary râles, pulmonary congestion on $x$-ray, third heart sound, increased jugular venous pressure); hypotension; shock; maximum heart rate, respiratory rate, minimum systolic blood pressure; different supraventricular and ventricular arrhythmias and conduction disturbances; site of infarct on electrocardiogram; maximum SGOT level within 18-48 $\mathrm{hr}$ of the onset of attack; heart volume on discharge ( $\mathrm{ml} / \mathrm{m}^{2}$ b.s.a.)
\end{abstract}

\section{Results}

During the follow-up period, I5I (32\%) of the 466 patients died. In Fig. I the results of the automatic interaction detector analyses are shown. A successive dichotomization based on the maximum respiratory rate registered during the stay in the coronary care unit, age, and left bundle-branch block (including hemiblock) proved most suitable for the identification of different prognostic groups. The number of patients, the mortality in each group, and the statistical differences between the mortality rates are shown. Patient groups with three-year mortalities ranging from 13 to 74 per cent were characterized. The low mortality rate was noted in patients with a maximum respiratory rate below 28 breaths/min during the period in the coronary care unit (mean 2.5 days) and who were less than 60 years of age. The poorest prognosis was registered in those who had shown a high respiratory rate and left bundle-branch block some time during the hospital stay. Sex and the remainder of the factors studied (Table) did not add significantly to the prognostic stratification when age, left bundlebranch block, and maximum respiratory rate were simultaneously considered.

Left bundle-branch block is the only qualitative factor appearing in the 'automatic interaction detector tree', the others are quantitative: age and respiratory rate. To illustrate the relation between the latter factors and three-year prognosis in patients with and without left bundle-branch block stepwise linear regression analyses were performed. The functions of the best dividing lines between

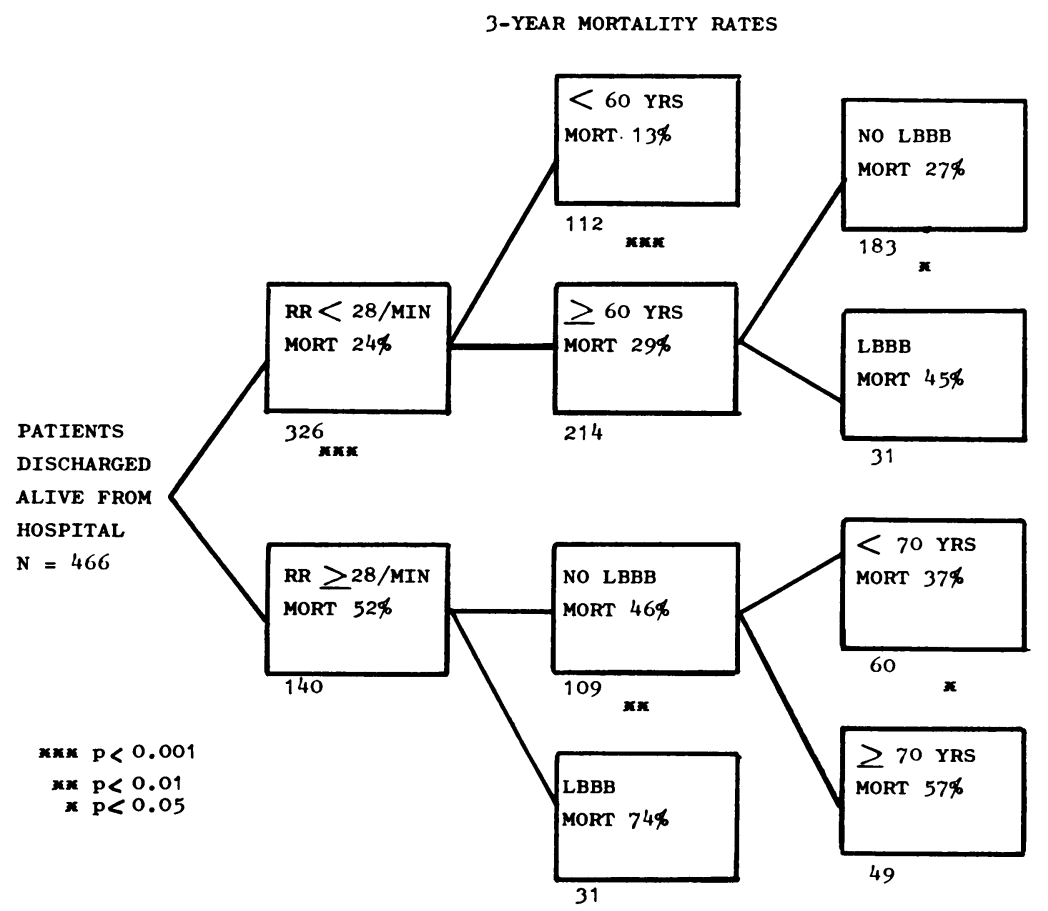

FIG. I A prognostic table for the first 3 years in survivors of acute myocardial infarction. $R R=$ maximum respiratory rate registered during the coronary care unit stay. $L B B B=$ left bundle-branch block (including hemiblock) some time during the hospital stay. The number of patients and the mortality in each group are shown. 


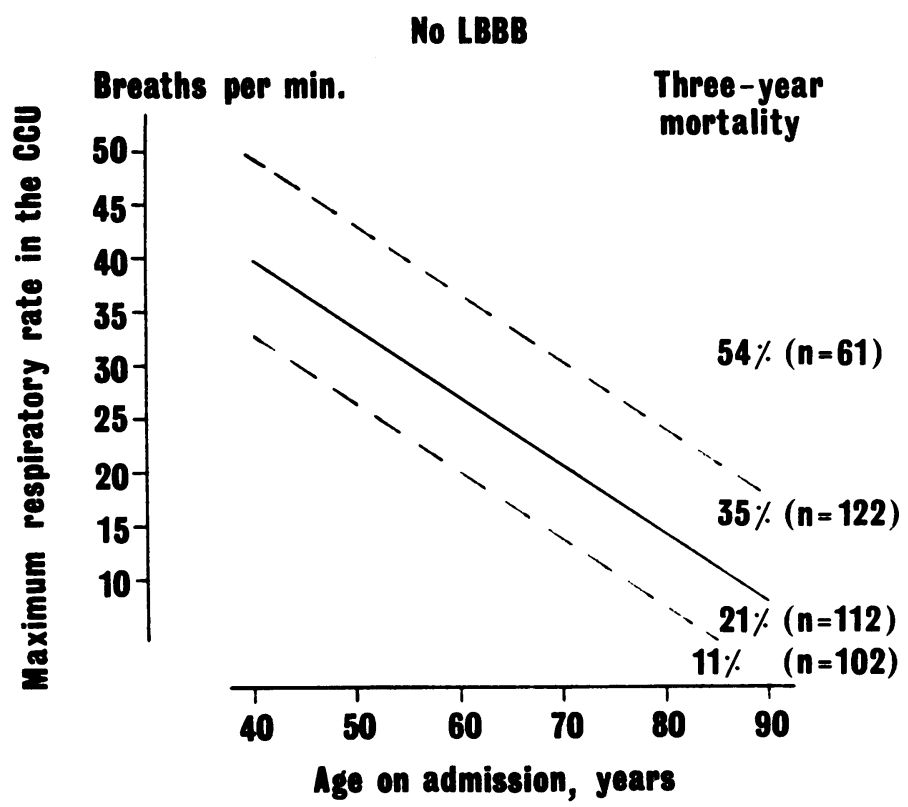

FIG. $2 A$ prognostic index for the first 3 years in survivors of acute myocardial infarction who have not shown left bundle-branch block during the hospital stay. For discussion, see text.

those patients who died during three years after the acute myocardial infarction and the survivors were calculated.

In patients without left bundle-branch block $(n=397)$ the mean value of the dependent variable (code: death within 3 years $=2$, survival $=1$ ) was I.27960 and the function of the best dividing line between survivors and deceased was:

I.27960 $=0.28830+0.01522 \times R R+0.00966 \times$ age,

where $R R=$ maximum respiratory rate recorded during the period in the coronary care unit. The continuous line in Fig. 2 corresponds to this function. The relation could also be described in terms of the most discriminatory respiratory rate at various ages: $R R=65.1314-0.6346 \times$ age.

The mortality among the 183 patients located above the line was 42 per cent and among those located below 16 per cent. In 69 per cent of the deceased patients and in 62 per cent of the survivors their fate was correctly predicted in the nomogram. There was no significant difference in predictability between patients with a reinfarction and those with a first acute myocardial infarct.

In the nomogram mortality rises with maximum respiratory rate and age. For further prognostic stratification the two dotted lines were added (Fig. 2). They were drawn so that only Io per cent of the deceased patients were classified below the lower line and ro per cent of the survivors above the upper line. In this way prognostic groups with three-year mortalities ranging from II to 54 per cent were identified.

In the group of patients $(n=69)$ who had shown left bundle-branch block (including hemiblock) some time during the hospital stay the function of the best dividing line between survivors and deceased patients was also calculated (Fig. 3):

$$
\mathrm{I} .56522=0.55 \mathrm{I} 28+0.02586 \times \mathrm{RR}+0.00433 \times \mathrm{age} \text {, }
$$

where 1.56522 is the mean value of the dependent variable coded as stated above. In terms of the most discriminatory respiratory rate at various ages the equation would be: $R R=39.2088-0.1674 \times$ age.

In 74 per cent of the survivors and in 54 per cent of the deceased patients the fate was correctly predicted in the nomogram. Two patient groups, with mortalities of 45 and 72 per cent, were characterized. When the patients who had shown left bundle-branch block were divided into two age groups, below and above 70 years of age, the three-year mortality was 55 per cent in the younger group ( $n=36$, mean age 63 years) compared with 57 per cent in the older group $(n=33$, mean age 77 years). In $4 \mathrm{I}(59 \%)$ of the 69 patients with left bundle-branch block the conduction disturbance was recorded in the last routine electrocardiogram taken before the patients' discharge from hospital. 


\section{LBBB}

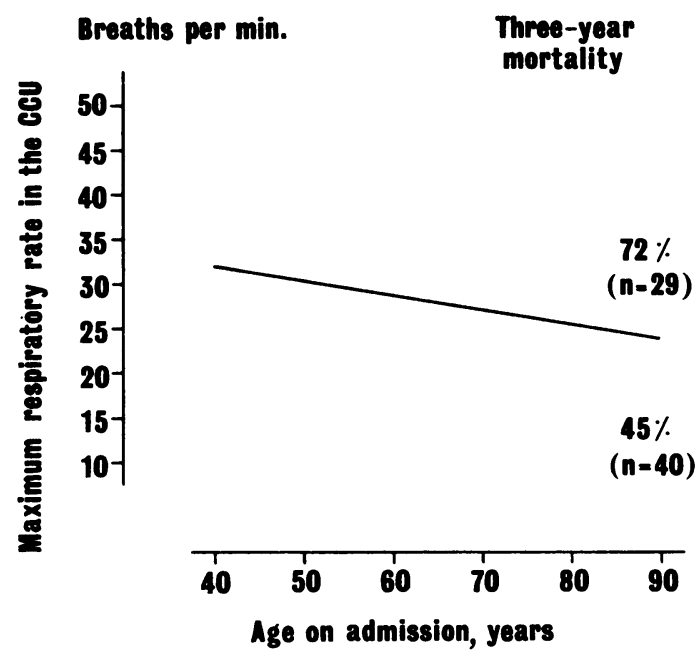

FIG. $3 A$ prognostic index for the first 3 years in survivors of acute myocardial infarction who have shown left bundle-branch block some time during the hospital stay.

Nineteen patients showed complete left bundlebranch block and 22 left anterior hemiblock. The three-year mortality was 47 and $4 \mathrm{I}$ per cent, respectively, in the two groups.

\section{Discussion}

The purpose of this study was to investigate the possibility of constructing an easily applicable index for the 3-year prognosis in survivors of acute myocardial infarction. An index based on the same patient group and relating to the first two years has been published previously (Helmers, 1973). This index included two factors only, age and maximum respiratory rate during the period in the coronary care unit. The prognostic indices of Peel et al. (1962) and Norris et al. (1970) included age as an important factor, too, but also other factors, several of which are related to the presence and degree of heart failure, e.g. pulmonary râles, pulmonary congestion on $x$-ray, and heart size. An increased respiratory rate was closely associated with a finding of pulmonary râles in the present patient group (Helmers, 1973). Pain, anxiety, acidosis, and pulmonary infections, all factors that may affect the respiratory rate are difficult to correct for and no attempt was made to do so. Instead it was considered important to keep the original patient group as intact as possible in the analyses. Maximum respiratory rate registered during the stay in the coronary care unit, age, and left bundle-branch block (including hemiblock) proved to be the best indicators of 3-year prognosis. Using these factors patient groups with mortalities ranging up to 74 per cent were identified. However, the difficulty in predicting the fate of the individual patient remained considerable. It was noted that in patients who had shown left bundle-branch block, mortality was very high also in relatively young age groups. In such patients there was no significant difference in mortality between those below and those above 70 years of age though the mean ages in the groups differed by 13 years. This finding once more demonstrates the great prognostic significance of left bundle-branch block in survivors of acute myocardial infarction.

As one of the purposes of a prognostic stratification is its use in therapeutic trials it may be an advantage if the factors included are accessible to treatment. In the present study, at least one, perhaps two of the three most important prognostic factors are beyond the reach of present therapeutic influence. Chronological age is definitely so, and though prophylactic pacemaking of patients with left bundle-branch block is possible it remains to be shown that such treatment offers any prognostic gain. The third factor, i.e. the maximum respiratory rate which was associated with the degree of cardiac failure during the hospital stay still remains. The importance of close observation for signs of heart failure and of adequate treatment, should such occur, does not need to be stressed.

In the present study it was as difficult to predict prognosis in patients with a reinfarction as in those with a first acute myocardial infarction. It has been suggested that these patient categories should not be compared in prognostic studies (Vedin, 1974). Relying on the present findings it is felt that the comparability between patients with a first infarct and those with reinfarct depends on the clinical factors used in the prognostic assessment. Apart from age, in a patient group with as wide an age range as the present one, the degree of cardiac damage and malfunction, as noted by electrocardiographic findings of left bundle-branch and respiratory rate, respectively, seems closely associated with the long-term prognosis both in patients surviving reinfarction and in those with a first acute myocardial infarct. In accordance with the findings of other authors (Norris and Mercer, 1973), it was noted that the presence of different supraventricular and ventricular arrhythmias during the period in the coronary care unit was not of great independent prognostic importance when the above factors were simultaneously considered. Should the target event be different, e.g. sudden death instead of 
death from all causes, other factors may prove of value when constructing a prognostic stratification. The number of patients included in the present study, however, was too limited to permit multivariate analyses with regard to different causes of death.

I thank Mr. Rein Maasing, for assistance with the statistics and the computer analyses. Financial support was given by the Swedish National Association against Heart and Chest Diseases.

\section{References}

Biörck, G., Lundman, T., Mogensen, L., and Orinius, E. (1969). Experiences from a coronary care unit. Archiv für klinische Medizin, 216, 242.

Helmers, C. (1973). Short and long-term prognostic indices in acute myocardial infarction. A study of 606 patients initially treated in a coronary care unit. Acta Medica Scandinavica, Suppl. 555.

Norris, R. M., Caughey, D. E., Deeming, L. W., Mercer, C. J., and Scott, P. J. (1970). Coronary prognostic index for predicting survival after recovery from acute myocardial infarction. Lancet, 2,485 .
Norris, R. M., and Mercer, C. J. (1973). Long-term prognosis following treatment in a coronary-care unit. Australian and New Zealand fournal of Medicine, 1,31 .

Osiris Program II, OS Users Manual (I97I). Inter-University Consortium for Political Research, The Institute for Social Research, The University of Michigan, Ann Arbor, Michigan.

Oxman, H. A., Connolly, D. C., Norbrega, F. T., Elveback, L. R., Titus, J. L., and Kurland, L. T. (1972). Factors influencing the subsequent prognosis of patients surviving their first myocardial infarction. Circulation, 45, Suppl. II, 200.

Peel, A. A. F., Semple, T., Wang, I., Lancaster, W. M., and Dall, J. L. G. (1962). A coronary prognostic index for grading the severity of infarction. British Heart fournal, 24,745 .

Sonquist, J. A., and Morgan, J. N. (1964). The Detection of Interaction Effects. Survey Research Center Monograph No. 35, Institute for Social Research, The University of Michigan, Ann Arbor, Michigan.

Vedin, J. A. (1974). Hjärtinfarkt i Götebørg 1968-70. Department of Medicine, Sahlgren's Hospital, Gothenburg.

Requests for reprints to Dr. C. Helmers, Department of Medicine, Serafimerlasarettet, Box I2780, I I2 83 Stockholm, Sweden. 